\title{
Multicriteria support of choosing a group decision
}

\author{
Andrzej Łodziński \\ Warsaw University of Life \\ Sciences ul. Nowoursynowska 159, \\ 02 - 776 Warszawa, Poland \\ Email: andrzej_lodzinski@sggw.pl
}

\begin{abstract}
The paper presents the method of a group decision making in a competitive environment. We deal with a group decision when the group of people with different preferences are to make one single decision. The group decision selection process is modeled with the use of multi-criteria optimization task. It is solved with the use of reference point method. This method is an interactive method in which every person specifies its requirements in the form of a reference point, expressing the desired values for its evaluation function. On the basis of the provided reference point, a scalar achievement function is built. Maximization of this function generates a solution of the multicriteria task. This solution is presented to every person for acceptance or as a basis for the modification of the reference point. The paper contains the example of application of the proposed method to support a group decision by three people with different preferences.
\end{abstract}

\section{INTRODUCTION}

The paper presents the method of a group decision making in a competitive environment. A group decision means that several people, whose interests are conflicting, are supposed to make one decision. One should conjoin divergent interest of all people, in order to arrive to a compromise solution for all. The aim of the selection decision is the best solution for the group, and not for individual members of that group. No solution is selected for a single person, it is looking for all persons in a group.

The selection process of a group decision can be modeled with the use of game theory [5], [6], [11], [14].

The process of a group decision making is modelled with the use of multi-criteria optimization with a vector evaluation function. Each coordinate of this vector is the value of decision evaluation function for each person. The decision selection is performed with the use of an interactive computer system. Each person provides his proposition of the decision result for his/her evaluation function. These propositions constitute parameters of the multi-criteria optimization task and that is then solved. Then, each person evaluates the solution. Each of them may agree to the obtained result or not. In the second case the person or persons provide a new value of the parameter - their new propositions and the problem is solved again for the new parameters. The selection process is not a one-time process, but an iterative process of learning about the decision making. The process of a group decision making is to support the members of a group to obtain as much as possible.

\section{MODELING OF A GROUP DECISION MAKING}

Our aim is to find an adequate group decision in a competitive case. The process of making a group decision is modeled by introducing a respective decision variable. Moreover, there are the s. c. decision evaluation functions, which constitute criteria evaluating the solution from the point of view of each person. Each person has its own evaluation criterion - its evaluation function. These functions are a measure of satisfaction of every person by a given solution; they evaluate a degree of achieving a goal by every person. The bigger value of the function means a bigger satisfaction, so every function is maximized. The basis for evaluation and selection of a group decision are all evaluation functions - the criteria for all persons.

The group decision selection problem is modeled as a multi-criteria optimization task:

$$
\max _{x}\left\{\left(f_{1}(x), f_{2}(x), \ldots, f_{k}(x)\right): \quad x \in X_{0}\right\}
$$

where: $1,2, \ldots, k$ - particular persons,

$X_{0} \subset R^{n}$ - the feasible set,

$x=\left(x_{1}, x_{2}, \ldots, x_{n}\right) \in X_{0}$ - a group decision,

$f_{i}: X_{0} \rightarrow R-$ the decision evaluation function

by a person $i, i=1,2, \ldots, k$.

Task (1) relies on finding such a feasible decision $x \in X_{0}$ for which $k$ evaluations attain the best possible values. There is a common restraining of decision which constitutes a solution.

The vector functions $f=\left(f_{1}, f_{2}, \ldots, f_{k}\right)$ defines the correspondence of any decision variable vector $x \in X_{0}$ and 
the respective evaluation vector $y=\left(y_{1}, \ldots, y_{k}\right)$. They measure the decision quality from the point of view of decision evaluation. Particular coordinates $y_{i}=f_{i}(x), \quad i=1, \ldots, k$ are scalar functions of decision evaluation for $i-t h$ person, $i=1,2, \ldots, k$. The image of the feasible set $X_{0}$ by the function $f$ constitutes a collection of achievable evaluation vectors $Y_{0}$.

Task (1) is formulated in the domain of evaluations, i.e. the following task is considered:

$$
\max _{x}\left\{\left(y_{1}, \ldots, y_{k}\right): \quad y \in Y_{0}\right\}
$$

where : $x \in X-$ a vector of decision variables, $y=\left(y_{1}, \ldots, y_{k}\right)-$ a vector of evaluations, particular coordinates $y_{i}$ representing the results of the decision $x$ for the person $i, i=1,2, \ldots, k$, $Y_{0}=f\left(X_{0}\right)-$ the set of achievable evaluation vectors.

The set of achievable evaluation vectors $Y_{0}$ is provided in a non-explicit way - through the set of feasible decisions $X_{0}$ and the model $f=\left(f_{1}, \ldots, f_{k}\right)$. In order to calculate the value $y$, a simulation of the respective model is necessary: $y=f(x), x \in X_{0}$.

The aim of task (1) is the aid in finding a decision that would be the most compromising for all persons.

\section{EQUITABLY EFFICIENT SOLUTION}

The solution in the selection decision process should satisfy certain properties that persons accept as reasonable. Namely, such a solution should be:

- $\quad$ an optimal solution in the sense of Pareto - i.e. such that you can not improve the solution for one person without worsening the solution for the other persons,

- $\quad$ symmetric solution - i.e. that it should not depend on the way the persons are numbered; as no one is more important that the others. Persons are treated in the same way in the sense that the solution does not depend on the name of person or on other factors specific to a given person,

- equalizing solution - that is, a vector that has less variation of coordinates of evaluation is preferred in comparison to a vector with the same sum of coordinates, but with a greater diversity of coordinates.

Any decision, that satisfies the above conditions is an equitably efficient decision. Hence, this Pareto-optimal decision satisfies additional conditions - anonymity and the axiom of equalizing solution.
The non-dominated results ( Pareto - optimal) are defined as follows:

$$
\hat{Y}_{0}=\left\{\hat{y} \in Y_{0}:(\hat{y}+\tilde{D}) \cap Y_{0}=\varnothing\right\}
$$

where: $\tilde{D}=D \backslash\{0\}$ - a positive cone without the top. As a positive cone, it can be adopted $\tilde{D}=R_{+}^{k}$. Appropriate acceptable decisions are specified in the decision space. The decision $\hat{x} \in X_{0}$ is called efficient decision (Pareto optimal), if the corresponding vector of evaluations $\hat{y}=f(\hat{x})$ is a non-dominated vector [7], 16], [17].

Finally, in the multi-criteria problem (1), which is used to select a group decision, the relation of preferences should satisfy additional properties: anonymity property and the property of equalizing solution.

The relation is called an anonymous relation if, for every vector $y=\left(y_{1}, y_{2}, \ldots, y_{k}\right) \in R^{k}$ and for any permutation $P$ of the set $\{1, \ldots, k\}$, the following property holds:

$$
\left(y_{P(1)}, y_{P(2)}, \ldots, y_{P(k)}\right) \approx\left(y_{1}, y_{2}, \ldots, y_{k}\right)
$$

No distinction is made between the results that differ in the arrangement of coordinates. Evaluation vectors having the same coordinates, but in a different order are identified and that is the anonymity property.

Moreover, the relation of preferences satisfies the axiom of equalizing transfer, if and only if the following condition is satisfied:

$$
\begin{aligned}
& \text { for the evaluation vector } \\
& y=\left(y_{1}, y_{2}, \ldots, y_{k}\right) \in R^{k}: \\
& \qquad y_{i^{\prime}}>y_{i^{\prime \prime}} \Rightarrow y-\varepsilon \cdot e_{i^{\prime}}+\varepsilon \cdot e_{i^{\prime \prime}} \succ y \\
& \text { for } 0<y_{i^{\prime}}-y_{i^{\prime \prime}}<\varepsilon
\end{aligned}
$$

Equalizing transfer is a slight deterioration of a better coordinate of evaluation vector and simultaneously improvement of a poorer coordinate. The resulting evaluation vector is strictly preferred in comparison to the initial evaluation vector. This is a structure of equalizing the evaluation vector with less diversity of coordinates is preferred in relation to the vector with the same sum of coordinates, but with their greater diversity.

Non-dominated vector satisfying the anonymity property and the axiom of equalizing transfer, is called equitably nondominated vector. The set of equitably non-dominated vectors is denoted by $\hat{Y}_{0 W}$. In the decision space, the equitably efficient decisions are specified. The decision $\hat{x} \in X_{0}$ is called equitably efficient decision, if the corresponding evaluation vector $\hat{y}=f(\hat{x})$ is an equitably 
non-dominated vector. The set of equitably efficient decisions is denoted by $\hat{X}_{0 W}$ [2], [9], [13].

The relation of equalizing domination can be expressed as the relation of inequality for cumulative, ordered evaluation vectors. This relation can be determined with the use of mapping $\bar{T}: R^{k} \rightarrow R^{k}$ that cumulates nondecreasing coordinates of evaluation vector.

The transformation $\bar{T}: R^{k} \rightarrow R^{k}$ is defined as follows:

$$
\bar{T}_{i}(y)=\sum_{l=1}^{i} T_{i}(y) \text { for } i=1,2, \ldots, k
$$

Define namely by $T(y)$ the vector with decreasing ordered coordinates of the vector $y$, i.e.. $T(y)=\left(T_{1}(y), T_{2}(y), \ldots, T_{k}(y)\right), \quad$ where $T_{1}(y) \leq T_{2}(y) \leq \ldots \leq T_{k}(y)$ and there is a permutation $P$ of the set $\{1, \ldots, k\}$, such that $T_{i}(y)=y_{P(i)}$ for $i=1, . ., k$.

The relation of equalizing domination $\succ_{e}$ is a simple vector domination for evaluation vectors with nondecreasing coordinates of evaluation vector [2], [9], [13].

The evaluation vector $y^{1}$ equitably dominates the vector $y^{2}$ if the following condition is satisfied:

$$
y^{1} \succ_{e} y^{2} \Leftrightarrow \bar{T}\left(y^{1}\right) \geq \bar{T}\left(y^{2}\right)
$$

Solving the problem of decision selection in the group decision process consists in determination of the equitably efficient decision which satisfies the preferences of every persons.

\section{SCALARING THE PROBLEM}

For determination of equitably efficient solutions of multi-criteria task (1), a specific multi-criteria task is solved. It is the task with the vector function of the cumulative, ordered evaluation vectors, i.e. the following task:

$$
\max _{y}\left\{\left(\bar{T}_{1}(y), \bar{T}_{2}(y), \ldots, \bar{T}_{k}(y)\right): \quad y \in Y_{0}\right\}
$$

where: $y=\left(y_{1}, y_{2}, \ldots, y_{k}\right)-$ an evaluation vector,

$$
\bar{T}(y)=\left(\bar{T}_{1}(y), \bar{T}_{2}(y), \ldots, \bar{T}_{k}(y)\right)
$$

a cumulative, ordered evaluation vector,

$Y_{0}$ - the set of achievable evaluation vectors.
Effective solution of multi-criteria optimization task (8) is an equitably efficient solution of the multi-criteria task (1).

To determine the solution of a multi-criteria task (8), the scalaring of this task with the scalaring function $s: Y_{0} \times \Omega \rightarrow R^{1}$ is solved:

$$
\max _{x}\left\{s(y, \bar{y}): x \in X_{o}\right\}
$$

where: $y=\left(y_{1}, y_{2}, \ldots, y_{k}\right)-$ an evaluation vector,

$$
\bar{y}=\left(\bar{y}_{1}, \bar{y}_{2}, \ldots, \bar{y}_{k}\right)-\text { a control parameters for }
$$
individual evaluations.

It is the task of single objective optimization with specially created scalaring function of two variables - the evaluation vector $y \in Y$ and control parameter $\bar{y} \in \Omega \subset R^{k}$; we have thus $s: Y_{0} \times \Omega \rightarrow R^{1}$. The parameter $\bar{y}=\left(\bar{y}_{1}, \bar{y}_{2}, \ldots, \bar{y}_{k}\right)$ is available to each person. That allows any person is capable to review the set of equitably efficient solutions.

The optimal solution of task (9) should be a solution of the multiple criteria task (8). Scalaring function should satisfy certain properties - the property of completeness and that of sufficiency. The property of sufficiency means that for each control parameter $\bar{y}$ the solution of the scalaring task is the equitably efficient solution, i.e. $\hat{y} \in \hat{Y}_{0 W}$. The property of completeness means, that by appropriate changes of parameter $\bar{y}$ any solution $\hat{y} \in \hat{Y}_{0 W}$ can be achieved. Such a function completely characterizes equitably efficient solutions. Inversely, each maximum of such a function is an equitably efficient solution. Each equitably efficient solutions can be obtained with appropriate value of control parameter $\bar{y}$.

Complete and sufficient parameterization of the set of equitably efficient solutions $\hat{Y}_{0 W}$ can be achieved, using the method of the reference point for the task (8). In this method the aspiration levels are applied as control parameters. Aspiration level is such value of the evaluation function that satisfies a given person.

The scalaring function defined in the method of reference point is as follows:

$$
\begin{aligned}
& s(y, \bar{y})=\min _{1 \leq i \leq k}\left(\bar{T}_{i}(y)-\bar{T}_{i}(\bar{y})_{i}\right) \\
& +\varepsilon \cdot \sum_{i=1}^{k}\left(\bar{T}_{i}(y)-\bar{T}_{i}(\bar{y})_{i}\right)
\end{aligned}
$$

where: $y=\left(y_{1}, y_{2}, \ldots, y_{k}\right)-$ an evaluation vector, $\bar{T}(y)=\left(\bar{T}_{1}(y), \bar{T}_{2}(y), \ldots, \bar{T}_{k}(y)\right)$ - a cumulative, ordered evaluation vector, 
$\bar{y}=\left(\bar{y}_{1}, \bar{y}_{2}, \ldots, \bar{y}_{k}\right)-$ a vector of aspiration levels,

$T(\bar{y})=\left(T_{1}(\bar{y}), T_{2}(\bar{y}), \ldots, T_{k}(\bar{y})\right)$ - a cumulative,

ordered vector of aspiration levels,

$\varepsilon-$ an arbitrary small, positive adjustment parameter.

Such scalaring function is called a function of achievement. The aim is to find a solution that approaches as close as possible the specific requirements - the aspiration levels [2], [7], [13].

Maximizing this function w. r. to $y$ determines the equitably efficient solution $\hat{y}$ and the equitably efficient decision $\hat{x}$. Note, the equitably efficient solution $\hat{x}$ depends on the aspiration level $\bar{y}$. A solution of the multicriteria optimization problem makes correspond of solution proposals of particular members of the group to the respective levels of aspiration.

\section{METHOD OF SUPPORTING THE GROUP DECISION}

The solution of the multi-criteria task (8) is a set of equitably efficient solutions. In order to solve a given problem it is necessary to pick one solution which will be evaluated by all persons. Due to the fact that the equitably efficient solution is a whole set of solutions, the persons perform the selection with the help of an interactive computer system. Such a system makes possible to have a guided overview of a whole set of solutions. The tool used to view this set of solutions is function (10). Maximum of this function depends on the parameters $\bar{y}_{i}, i=1,2, \ldots, k$, which are applied by all persons. In the reference point method each person expresses its preferences by specification, with the aid of his/her evaluation function, of such a value that would be fully satisfactory. That is the value of the aspiration level for his/her evaluation function. For any stage of the selection process the persons may provide different aspiration levels. Such levels of aspiration constitute steering parameters of the scalarization function. On this basis the task is solved and the system proposes the solution corresponding to the current values of those parameters - for further analysis.

The method of supporting the group decision is the following:

1. Iterative algorithm - propositions of particular decision.

1.1. Interaction with the system - each person provides his/her own proposition of the decision for its evaluation function as his/her level of aspiration $\bar{y}_{i}, i=1,2, \ldots, k$.

1.2. Calculations - computing particular values from the equitably efficient solution $\hat{y}=\left(\hat{y}_{1}, \hat{y}_{2}, \ldots, \hat{y}_{k}\right) \in \hat{Y}_{0 W}$ and the equitably efficient decision $\hat{x}=\left(\hat{x}_{1}, \hat{x}_{2}, \ldots, \hat{x}_{k}\right) \in \hat{X}_{0 W}$.

1.3. Evaluation of the obtained solution - each person may accept the solution or not. In the second case each person provides his/her new proposition and provides a constant value of his/her level of aspiration $\bar{y}_{i}, i=1,2, . ., k$ and another equitably efficient solution is set out. (Return to sub-point 1.2).

2. Establishing the decision, when the decision fulfills the requirements of all persons.

This is not a single optimization act but a dynamic process of looking for solutions, during which the persons learn and may change their preferences. Comparing the result of the decision $\hat{y}_{i}, i=1,2, \ldots, k$ with the aspiration point $\bar{y}_{i}, i=1,2, \ldots, k$, each person finds what is not achievable and how his/her proposition $\bar{y}_{i}, i=1,2, \ldots, k$ is far from a possible solution $\hat{y}_{i}, i=1,2, \ldots, k$. This allows for a proper modification of their own propositions - with regard to their own levels of aspiration. These levels of aspirations are specified adaptively in the process of teaching. This process finishes when such decisions are found, which allow to fulfill the aspirations of persons in a maximum possible degree.

Method of supporting the group decision is presented at diagram 1.

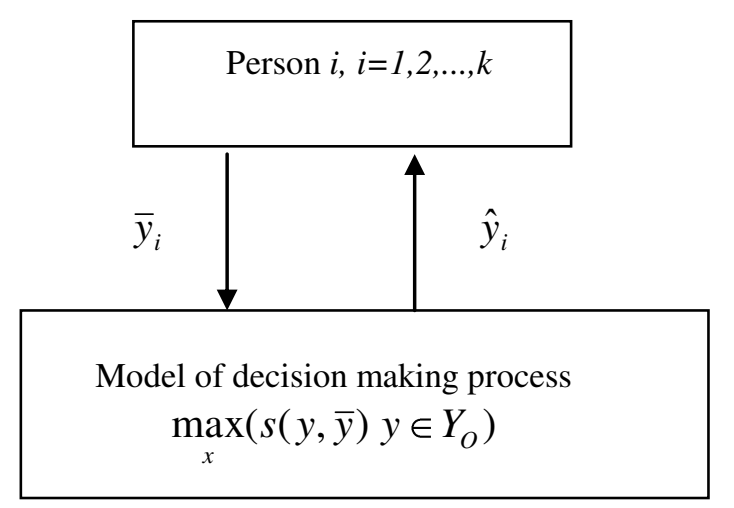

Fig. 1. Method of supporting the group decision making

Such a manner of making decisions does not impose any strict scenario and allows for the possibility of modifying the preferences for every person in the decision making process. Persons learn during the selection process about the decision making problem. The persons may check the results of every allowed proposition. All members of a group have an equal part in the decision making process. They all have an equal possibility for eventual changes of their preferences. The computer will not replace people in the decision making 
process; the whole process of selecting a decision is guided by all persons.

\section{EXAMPLE}

To illustrate the support of the group decision making the following example is presented - selection of a group decision by three persons [3].

The problem of selecting the decision is the following: 1,2,3 -persons,

$$
\begin{aligned}
& X_{0}=\left\{x \in R^{4}: 2 \cdot x_{1}+x_{2}+4 \cdot x_{3}+3 \cdot x_{4} \leq 60,\right. \\
& 3 \cdot \mathrm{x}_{1}+4 \cdot x_{2}+x_{3}+2 \cdot x_{4} \leq 60 \\
& \left.x_{1} \geq 0, \mathrm{x}_{2} \geq 0, \mathrm{x}_{3} \geq 0, \mathrm{x}_{4} \geq 0\right\}
\end{aligned}
$$

the feasible set,

$x=\left(x_{1}, x_{2}, x_{3}, x_{4}\right) \in X_{0}$ - a group decision,

$f_{1}(x)=3 \cdot x_{1}+x_{2}+2 \cdot x_{3}+x_{4}-$ the decision evaluation function by person 1 ,

$f_{2}(x)=x_{1}-x_{2}+2 \cdot x_{3}+4 \cdot x_{4}-$ the decision evaluation function by person 2 ,

$f_{3}(x)=-x_{1}+5 \cdot x_{2}+x_{3}+2 \cdot x_{4}-$ the decision evaluation function by person 3 ,

The problem of selection of a group decision is expressed in the form of multi-criteria optimization task with three evaluation functions:

$$
\begin{aligned}
& \max _{x}\left\{\left(3 \cdot x_{1}+x_{2}+2 \cdot x_{3}+x_{4},\right.\right. \\
& x_{1}-x_{2}+2 \cdot x_{3}+4 \cdot x_{4}, \\
& \left.\left.-x_{1}+5 \cdot x_{2}+x_{3}+2 \cdot x_{4}\right): \quad x \in X_{0}\right\}
\end{aligned}
$$

where: $X_{0}$ - the feasible set, $x=\left(x_{1}, x_{2}, x_{3}, x_{4}\right) \in X_{0}$ - a group decision

To select the solutions of (11), the reference point method is used for the task with cumulated coordinates of the evaluation vector ordered in a non decreasing manner.

The first step of the vector analysis is to use the onecriteria optimization for evaluation function of every person separately. As a result there is the so-called matrix of goal realization including the values of each criterion, received by solving one of the three one-criteria problems. This matrix allows for evaluation of the scope of changes of particular evaluation function on the allowed set; it provides a certain information about the conflict of the evaluation functions. Matrix of goal realizations generates the utopia vector that represents the best values of each separate criterion.
Table 1. Matrix of goal realization with the utopia vector.

\begin{tabular}{|ll|ccc|}
\hline \multicolumn{2}{|c|}{$\begin{array}{c}\text { Optimization } \\
\text { criterion }\end{array}$} & $\hat{y} 1$ & $\hat{y} 2$ & $\hat{y} 3$ \\
\hline Person's Evaluation 1 & $y 1$ & 66 & 30 & -12 \\
Person's Evaluation 2 & $y 2$ & 20 & 80 & 40 \\
Person's Evaluation 3 & $y 3$ & 15 & -15 & 75 \\
\hline Utopia vector & & 66 & 80 & 75 \\
\hline
\end{tabular}

When analyzing the table 1 it might be observed that the biggest selection possibilities has person 2, lower - person 3

\begin{tabular}{|c|c|c|c|}
\hline Iteration & Pers. 1 & Pers2 & Pers. 3 \\
\hline $\begin{array}{l}\text { Aspiration point } \bar{y} \\
\text { Solution } \hat{y}\end{array}$ & $\begin{array}{l}66 \\
24\end{array}$ & $\begin{array}{l}80 \\
66\end{array}$ & $\begin{array}{l}75 \\
66\end{array}$ \\
\hline $\begin{array}{l}\text { Aspiration point } \bar{y} \\
\text { Solution } \hat{y}\end{array}$ & $\begin{array}{l}55 \\
26,76\end{array}$ & $\begin{array}{l}65 \\
65\end{array}$ & $\begin{array}{l}60 \\
60\end{array}$ \\
\hline $\begin{array}{ll}3 & \text { Aspiration point } \bar{y} \\
& \text { Solution } \hat{y}\end{array}$ & $\begin{array}{l}50 \\
30,17\end{array}$ & $\begin{array}{l}60 \\
60\end{array}$ & $\begin{array}{l}55 \\
55\end{array}$ \\
\hline $\begin{array}{l}\text { Aspiration point } \bar{y} \\
\text { Solution } \hat{y}\end{array}$ & $\begin{array}{l}48 \\
31,54\end{array}$ & $\begin{array}{l}58 \\
60\end{array}$ & $\begin{array}{l}53 \\
55\end{array}$ \\
\hline $\begin{array}{l}\text { Aspiration point } \bar{y} \\
\text { Solution } \hat{y}\end{array}$ & $\begin{array}{l}45 \\
33,59\end{array}$ & $\begin{array}{l}55 \\
55\end{array}$ & $\begin{array}{l}50 \\
50\end{array}$ \\
\hline $\begin{array}{l}\text { Aspiration point } \bar{y} \\
\text { Solution } \hat{y}\end{array}$ & $\begin{array}{l}43 \\
34,5\end{array}$ & $\begin{array}{l}53 \\
53\end{array}$ & $\begin{array}{l}48 \\
48\end{array}$ \\
\hline
\end{tabular}
and the lowest one - person 1.

People in the group do control the process by means of aspiration levels. The multi-criteria analysis is presented in table 2 .

Table 2. Interactive analysis of looking for solutions.

At the beginning of the analysis every person specifies its preferences as the aspiration point equal to the utopia vector coordinate. The obtained solution prefers by person 2 and person 3 and is too small for person 1. The group wants to improve the solution. Therefore, all the people in the group decrease their requirements in the next iteration. One obtains a slight improvement for person 1 and deterioration for person 2 and person 3, but the group wants to improve the solution for person 1 . In subsequent iterations all individuals reduce their requirements and improve the value obtained for the assessment of persons 1 , at the cost of two other person. For iterations 5 and 6 the following decisions are found:

$\hat{x}^{5}=(3,78 ; 5,12 ; 2,02 ; 13,07)$

and $\hat{x}^{6}=(4,27 ; 5,07 ; 2,41 ; 12,24)$. The analysis reveals that there is deep influence of person 2 and 3 on the solution; however, for person 1 it is far less significant. 
The final selection of the specific solution depends on the preferences of all persons. The presented example shows that the method allows the persons to learn about their decisionmaking possibilities. The search for compromise for everyone is continued in this method.

\section{CONCLUSION}

The paper presents the method of supporting the group decision making. The selection of decision is performed by solving the multi-objective task according to the optimization criteria. This method is characterized by:

- the use of information about everyone's preferences in the form of aspiration points - values of goal function that are fully satisfactory to them and the optimal option of the scalar achievement function in order to organize the interactions with all persons,

- the assumption that the preferences of persons are not completely fixed and they may change during the decision making process.

Reference point method applied to the of multi-criteria problem indicates a solution which would be suited to the preferences of all individuals in the group.

The participation of any members of the group in the decision making implies acceptation of a final choice. In such a course of action one does not replace people in decision making. The whole process of decision making is guided by all persons.

\section{REFERENCES}

[1] U. Chevaleyre,. J. Endriss, M. Lang, and N. Maudet "A Short Introduction to Computational Social Choice”. Proc. SOFSEM-2007, Springer-Verlag, 2007.

[2] M. Kostreva., W. Ogryczak. and A., P., Wierzbicki "Equitable Aggregation and Multiple Criteria Analysis". European Journal of Operational Research, vol. 122., 2004.

[3] S. Krawczyl "Mathematical analysis of the situation of decisionmaking". Warsaw ( in polish), 1990.

[4] L. Kruś "On some Procedures Supporting Multicriteria Cooperative Decisions". Foundations of Computing and Decision 33, (3), 2008.

[5] L. Kruś "Multi-criteria decision cooperative. Computer-aided methods", Warsaw, 2011.

[6] D. Luce., H. Raiffa. "Games and decisions". (in polish) PWN, Warsaw, 1996.

[7] A. Lewandowski A. and A., P. Wierzbicki eds. "Aspiration Based Decision Support Systems". Lecture Notes in Economics and Mathematical Systems. Vol. 331, Springer-Verlag, Berlin-Heidelberg, 1989.

[8] J. Lu, G Zhang and D. Ruan "Multi-objective group decision making: methods, software and applications with fuzzy set techniques" dl.acm.org. 2007.

[9] A. Łodziński A. „The reference point method applied to decision selection in the process of bilateral negotiations" Metody Ilościowe w Badaniach Ekonomicznych / Quantitative Methods in Economics, Warsaw, 2014.

[10] A. Łodziński A. "Interactive method of selection decisions under risk" Zarządzanie a Inżynieria Produkcji Management versus Production Engineering, (in polish) Kraków 2013. 